\title{
Notiz zur Chemie \\ der Bikarbonate des Calciums und Magnesiums.
}

\author{
Von \\ C. Kippenberger.
}

In einer Arbeit, betitelt: „Über die Löslichkeit der Bikarbonate des Calciums und Magnesiums" geben Treadwelt und Reuter ${ }^{1}$ Mitteilungen über Untersuchungen, welche sich aufser auf die in dem Titel ausgesprochenen Versuche auch auf die Existenz der genannten Verbindungen in wässerigen Lösungen beziehen und kommen zu Resultaten, die in ihrem wesentlichen Teile am Schlusse der Arbeit in drei Thesen zusammengestellt sind.

Danach soll:

„Magnesiumbikarbonat nicht ohne grofsen Überschufs an freier, in Wasser gelöster Kohlensäure existieren", und citieren die genannten Autoren die diesbezüglichen Litteraturangaben dahin, dals:

Cossa und besonders Krppenberger die Existenz des Mayr. nesiumbikaŗbonats annehmen. ${ }^{2}$

In der von Treadweil und Recter citierten Arbeit von $\mathrm{mir}^{3}$ habe ich mich S. 188 wörtlich geäufsert:

„Die leichte Zersetzungsfähigkeit der Doppelverbindung beider Sesquikarbonate in neutrales Nagnesiumkarbonat und Rückbildung ron Alkalikarbonat begründet sich durch die Unbeständigkeit aller kohlensauren Verbindungen des Magnesiums, welche mehr Kohlensäure enthalten, als zur Bildung von Monokarbonat entspricht, indem sich Magnesiumsesquikarbonat und ebenso schnell Magnesiumbikarbonat in Magnesiumkarbonat und

1 Z. anorg. Chem. (1898) 17, 170-204.

2 S. 202.

${ }^{3}$ Z. anorq. Chem. (1894) 6, 177-194.

Z. anorg. Chein. XritI. 
freie Kohlensäure dissoziieren. Nur wenn gröfsere Mengen völlig freier Kohlensäure vorhanden sind, vermag die doppeltkohlensaure Verbindung des Magnesiums längere Zeit beständig zu bleiben, und ist die Menge der freien Kohlensäure eine ziemlich grofse, so kann man in diesem Falle von der Gegenwart eines Sesquikarbonats natürlich nicht sprechen; überwiegt aber die vorhandene freie Kohlensäure nur um ein geringes diejenige Menge Kohlensäure, welche zur Bildung ron Bikarbonat der Berechnung nach entspricht, so beginnt schon sofort eine Dissoziation in Sesquikarbonat bezüglich Karbonat und freie Kohlensäure einzutreten, und ich möchte behaupten, dafs es eine Existenz eines Magnesiumbikarbonats ohne freie Kohlensäure, also die eines idealen $\mathrm{MgCO}_{3} . \mathrm{CO}_{2}$ oder $\mathrm{MgH}_{2}\left(\mathrm{CO}_{3}\right)_{2}$ überhaupt nicht giebt."

Das von mir im Jahre 1894 genommene Patent Nr. 78767: „Verfahren zur Darstellung reinen, krystallinischen, neutralen Magnesiumkarbonats," gründet sich neben verschiedenen anderen chemischen Thatsachen (Unlöslichkeit von Calciumkarbonat, Eisenhydroxyd u. s. w. unter gleichen Versuchsbedingungen u. s. w.) auf die vermehrte Löslichkeit von Magnesiumkarbonat in wässeriger Alkalibikarbonatlösung und Zersetzung des dabei gebildeten Magnesiumsesquikarbonats infolge Dissoziation unter Regenerierung von Magnesiumkarbonat; es ist dies auch nochmals in den Zusätzen ausgesprochen, welche ich hierzu in einer Arbeit, betitelt: „Über die chemischen Vorgänge bei der Einwirkung einzelner wasserlöslicher Salze auf die Karbonate der alkalischen Erden und auf Magnesiumkarbonat" gegeben habe, wobei ich von fein verteiltem frischgeglühtem - Magnesiumhydroxyd-, bezw. magnesiumoxydhaltigem Präparate ausging. Ich habe mich an genannter Stelle (S. 255) wörtlich ausgedrückt:

„Es tritt zunächst Magnesiumkarbonat mit dem Alkalibikarbonat zu Sesquikarbonat in Wirkung; durch den grolsen Überschuls des Magnesiumkarbonats geht die Reaktion aber noch weiter und es wird - wie in der Arbeit vorher beschrieben — schliefslich ein Teil des Alkalisesquikarbonats in Monokarbonat verwandelt; einerseits hierdurch, andererseits durch das beständige Schütteln der Flüssigkeit und endlich durch die Massenwirkung des Magnesiumkarbonats wird das gebildete und leicht dissoziierende Mag-

1 Ber. pharm. Gesellschaft (R. Gänmnen's Verlag, Berlin) 1895, 245-257. 
nesiumsesquikarbonat in Monokarbonat verwandelt, fällt als solches in kleinkrystallinischem und dadurch passivem Zustande aus, worauf ein neuer Teil des aktiven Magnesiumkarbonats in Lösung geht, und der Vorgang sich nun wiederholt."

Auch in meiner Arbeit: „Zur titrimetrischen Trennung von Hydroxyden und Karbonaten, Bikarbonaten und Karbonaten der Alkalien, alkalischen Erden und des Magnesiums" befinden sich an verschiedenen Stellen Angabien über die Bikarbonate des Magnesiums und des Calciums, aus denen ebenso wie aus den oben citierten Stellen recht deutlich hervorgeht, dals ich genau das Gleiche ausgesprochen habe, was Treanweili und Reuter auf Grund der erneuten Versuche über die Existenz der Bikarbonate des Magnesiums behaupten und ich mich somit gegen die Angabe ron TREADweLL und Revter, dafs ich die Existenz des Magnesiumbikarbonats annehme, entschieden verwahren mufs, vielmehr das Resultat der Untersuchungen von TrEadweLL und REUTER als eine Bestätigung und Ergänzung meiner bereits im Jahre 1894 gegebenen Mitteilungen ansehe. $^{2}$

Seite 192 behaupten sodann Treadwelu und Reuter:

„Aus Kippenberger's Versuchen geht hervor, dals Calcium. karbonat in konzentrierter Kochsalzlösung ca. dreimal löslicher ist, als in Wasser. Diese gröfsere Löslichkeit ist wahrscheinlich bedingt durch die Bildung von Doppelsalzen."

Auch diese Angabe von Treadwelil und Reuter mufs ich richtig stellen:

Die genannten Autoren geben nicht an, wo sich meine Angaben in der Litteratur befinden, aus denen sie diese Schlüsse ziehen. Ich citiere deshalb aus meiner Arbeit: „Beiträge zur Kenntnis der Bildung kohlensaurer Salze in Trink- und Mineralwässern" (S. 7) des im Buchhandel befindlichen Sonderabdrucks, ${ }^{3}$ wörtlich folgende Stelle:

„Bei der Einwirkung von Calciumkarbonat scheint allerdings

1 Zeitschr, angew. Chem. 1894, Heft 16.

2 Auch die Übertragung meiner Angaben über Magnesiumbikarbonat auf die praktischen Verhältnisse findet sich in einer meiner citierten Arbeiten gelegentlich der Besprechung der Hunyadi-Janos Bittersalzquelle (,Forschungsber. über Lebensmittel u. §. w." 1894, Heft 12, S. 488).

8 „Forschungsber. über Lebensmittel und ihre Beziehungen zur Hygiene, forensische Chemie u. s. w." (WolfF's Verlag, München) 1894, Heft 7, S. 227 bis 237, und Heft 8, S. 263-275; siehe speziell S. 231. 
in der Kälte eine allmähliche geringe Umsetzung mit dem Alkalisalz stattzufinden, was bei der Anwendung von Alkalisulfat und Alkalinitrat noch deutlicher hervortritt als bei dem Chlorid."

Später habe ich in der oben citierten Arbeit in den Berichten der pharmaceut. Gesellschaft, Berlin, meine Ansicht ausführlich dahin lautend klargelegt, dafs bei diesen Umsetzungen bezw. bei der vermehrten Löslichkeit der Karbonate die hydrolytische Dissoziation der angewendeten Salze eine wesentliche Rolle spielt; ${ }^{1}$ ich rerweise auf die S. 252 der genannten Berichte speziell für den vorliegenden Fall gegebenen Darlegungen. Es geht daraus deutlich hervor, dafs in der Kälte und bei Anwendung frisch gefällten Calciumkarbonats zunächst eine Vermehrung der Löslichkeit des Calciumkarbonats durch Kochsalz hauptsächlich in Form des Calciumchlorids eintritt, und diese Umsetzung durch den Zerfall eines Teiles der Salze hervorgerufen wird; ich will dem hinzufügen, dafs Wärme oder auch die langandauernde Einwirkung der Salze in wässeriger Lösung unter öfterem Umschwenken derselben voraussichtlich weitere Umsetzungen zu Gunsten der Bildung von Calciumkarbonat hervorrufen können.

In diesen Arbeiten sowohl, wie auch in der oben citierten Arbeit ${ }^{2}$ befinden sich aufserdem Mitteilungen, mit welchen ich die Existenz des Calciumbikarbonats ausgesprochen und dabei auf Grund experimenteller Untersuchungen die Existenz, bezw. Beständigkeit der Sesquikarbonate der alkalischen Erden als unwahrscheinlich angesehen habe. ${ }^{3}$ -

${ }^{1}$ Ich will nicht versäumen, darauf hinzuweisen, dal's die hydrolytische Dissoziation der Salze von einigen Chemikern entweder ganz bestritten oder deren Einflufs als unbedeutend angesehen wird. Aufser den an genannten Stellen gegebenen Erörterungen habe ich mich hierüber unter anderem auch in einer Abhandlung in der Chem.-Ztg. 1895, 1269 ausgesprochen. Inzwischen sind von G. Pratner ganz ähnliche Versuche wie die an letztgenannter Stelle von mir angegebenen Untersuchungen durchgeführt, und deren Resultate in genau derselben Weise wie von mir erklärt worden (Elektrochem. Zeitschr. 189S). Da Puatrer gänzlich unabhängig von mir und mit Übersehung meiner Publikationen gearbeitet hat, so bieten dessen Resultate eine wesentliche Stütze und Ergänzung meiner Darlegungen.

ZZeitschr. angew. Chem. 1894, Heft 16.

${ }^{3}$ Das als existenzfäbig bezeichnete Bikarbonat der alkalischen Erden wird in der wässerigen Lösung natürlich bis zu einem gewissen Grade, ebenso wic andere Salze der alkalischen Erden, hydrolytisch gespalten sein, und diese Spaltung wird bei den Bikarbonaten in gröfserem Malse stattfinden als bei den anderen Salzen, z. B. den Chloriden, da die Kohlensäure eine schwache Säure 
Meine Untersuchungen sind speziell zur Klarlegung der bei den Trink- und Mineralwässern eintretenden Verhältnisse unternommen worden; es spielt bei der Auslaugung der Erdschicht durch das Wasser nicht allein die Löslichkeit der Salze als solche in einem mit Kohlensäure mehr oder weniger geschwängerten Wasser eine Rolle, sondern auch die Dissoziation der im Wasser allmählich sich lösenden Verbindungen übt auf Auslösung und Umsetzung in wesentlichem Unfange einen Einflufs aus.

ist. Immerhin ist aber das Spannungsverhältnis zwischen Base und Säure bei diesen Bikarbonoten ein derartiges, dafs man von einer Bikarbonatlösung sprechen kann.

Kairo, Chemisches Laboratorium der mediz. und pharmaceut. Hochschule, den 16. August 1898.

Bei der Redaktion eingegangen am 19 August 1898. 